\title{
Distributed optimal control problems for phase field systems with singular potential
}

\author{
Pierluigi Colli, Gianni Gilardi, Gabriela Marinoschi and Elisabetta \\ Rocca
}

\begin{abstract}
In this paper we review some results obtained for a distributed control problem regarding a class of phase field systems of Caginalp type with logarithmic potential. The aim of the control problem is forcing the location of the diffuse interface to be as close as possible to a prescribed set. However, due to some discontinuity in the cost functional, we have to regularize it and solve the related control problem for the approximation. We discuss the necessary optimality conditions.
\end{abstract}

\section{Introduction}

This note is aimed to review and discuss the results contained in [17], then it deals with a distributed control problem for the phase field system (see [12] and [11])

$$
\partial_{t} \vartheta-\Delta \vartheta+\partial_{t} \varphi=f, \quad \partial_{t} \varphi-\Delta \varphi+\mathcal{W}^{\prime}(\varphi)=\vartheta
$$

in $Q:=(0, T) \times \Omega$, where $\Omega$ is a bounded domain in space and $T$ is some final time. The variables $\vartheta$ and $\varphi$ denote the (relative) temperature and the order parameter, respectively. Moreover, $f$ stands for a source term and $\mathcal{W}^{\prime}$

Key Words: Phase field systems, singular potentials, optimal control, optimality conditions, adjoint state system

2010 Mathematics Subject Classification: 49J20, 35K55, 80A22.

Received: March, 2017.

Revised: May, 2017.

Accepted: June, 2017. 
DISTRIBUTED OPTIMAL CONTROL PROBLEMS

represents the derivative of a double-well potential $\mathcal{W}$. The initial conditions $\vartheta(0)=\vartheta_{0}$ and $\varphi(0)=\varphi_{0}$ and suitable boundary conditions must complement the system of equations in (1). Let us fix homogeneous Dirichlet boundary conditions for $\vartheta$ (we recall that $\vartheta$ is a relative temperature so that 0 can play as a reference value) and no flux boundary conditions for $\varphi$, namely

$$
\vartheta=0, \quad \partial_{n} \varphi=0
$$

on $\Sigma:=(0, T) \times \Gamma$. Here, $\Gamma$ is the boundary of $\Omega$ and $\partial_{n}$ denotes the outward normal derivative. The condition for $\varphi$ is very common in the literature since it is the natural one for the phase variable.

Let us set an optimal control problem for the resulting initial-boundary state system. The control is considered as part of the forcing term $f$ in the right-hand side of the first equation (1) and it is allowed to act only on a part $\Omega_{\text {act }} \subset \Omega$. Hence, we can take $f(t, x)=m(x) u(t, x)$, where $m$ is in principle the characteristic function of $\Omega_{\text {act }}$ and $u$ is the control. The state system takes the form

$$
\begin{array}{cc}
\partial_{t} \vartheta-\Delta \vartheta+\partial_{t} \varphi=m u & \text { in } Q, \\
\partial_{t} \varphi-\Delta \varphi+\mathcal{W}^{\prime}(\varphi)=\vartheta & \text { in } Q, \\
\vartheta=0 \quad \text { and } \quad \partial_{n} \varphi=0 & \text { on } \Sigma, \\
\vartheta(0)=\vartheta_{0} \quad \text { and } \quad \varphi(0)=\varphi_{0} & \text { on } \Omega,
\end{array}
$$

and the control $u$ is supposed to vary in some control box $\mathcal{U}_{a d}$. Our aim is forcing the location of the diffuse interface of $\varphi$, i.e., of the set $\{-\varepsilon \leq \varphi \leq \varepsilon\}$ for some given $\varepsilon>0$, to closely approach a prescribed set $E \subset Q$. Then, if we denote by $\chi_{E}$ the characteristic function of $E$ and by $g$ the characteristic function of the interval $[-\varepsilon, \varepsilon]$, the cost functional

$$
J_{0}(u):=\frac{1}{2} \int_{Q}\left(g(\varphi)-\chi_{E}\right)^{2},
$$

where $(\vartheta, \varphi)$ is the state corresponding to $u$, can be considered. More generally, we could take a cost functional being the sum of two contributions:

$$
\mathcal{J}(u):=\frac{1}{2} \int_{Q}\left(g(\varphi)-\chi_{E}\right)^{2}+\frac{\kappa}{2} \int_{Q}\left(\vartheta-\vartheta_{Q}\right)^{2},
$$

where the second is based on a given reference temperature $\vartheta_{Q} \in L^{2}(Q)$ and some proportionality constant $\kappa \geq 0$. With the choice (8) the optimal control (provided it exists) should balance the closeness of the set $\{-\varepsilon \leq \varphi \leq \varepsilon\}$ to $E$ and the smallness of the quantity $\left|\vartheta-\vartheta_{Q}\right|^{2}$, depending on the value of the coefficient $\kappa$. 
DISTRIBUTED OPTIMAL CONTROL PROBLEMS

At this point, we claim that the related problem would be rather difficult for every reasonable control box $\mathcal{U}_{a d}$. As this is essentially due to the discontinuous character of $g$, it turns out that the characteristic function $g$ can be replaced by a continuous approximation of it (still denoted by $g$ ) or even, in order to generalize the problem, by any continuous function on $\mathbb{R}$ satisfying some growth condition that makes the cost functional meaningful for every admissible control $u$. Moreover, we can substitute $\chi_{E}$ with a more general given function.

Anyhow, the difficulty due to the presence of the nonlinearity $\mathcal{W}^{\prime}(\varphi)$ in (4) remains. Concerning the double-well potential $\mathcal{W}$, the typical example is provided by the classical regular potential (cf. [12])

$$
\mathcal{W}_{\text {reg }}(r)=\frac{1}{4}\left(r^{2}-1\right)^{2}, \quad r \in \mathbb{R}
$$

Of course, other choices of $\mathcal{W}$ are possible. A thermodynamically significant example is given by the so-called logarithmic double-well potential [7, 8, 38], namely

$$
\mathcal{W}_{l o g}(r)=((1+r) \ln (1+r)+(1-r) \ln (1-r))+c\left(1-r^{2}\right), \quad r \in(-1,1),
$$

where $c>0$ is taken large enough in order that $\mathcal{W}_{l o g}$ may actually exhibit two wells, with a local maximum at $r=0$. More generally, the potential $\mathcal{W}$ can be assumed to be the sum $\mathcal{W}=\widehat{\beta}+\widehat{\pi}$, for some convex and lower semicontinuous function $\widehat{\beta}$ that is allowed to take the value $+\infty$ as well, and for a smooth concave perturbation $\widehat{\pi}$. In such a case, $\widehat{\beta}$ is supposed to be proper (not identically $+\infty$ ) so that its subdifferential is well defined and can replace the derivative which might not exist. In this respect, an interesting example is the so-called double obstacle potential (see [35])

$$
\mathcal{W}_{o b s}(r)=I_{[-1,1]}(r)+c\left(1-r^{2}\right), \quad r \in \mathbb{R},
$$

where $I_{[-1,1]}$ denotes the indicator function of the set $[-1,1]$, which takes the values 0 in $[-1,1]$ and $+\infty$ outside. Let us point out that, in these cases, the equation (4) becomes

$$
\partial_{t} \varphi-\Delta \varphi+\beta(\varphi)+\pi(\varphi) \ni \vartheta \quad \text { in } Q
$$

and it reads as differential inclusion.

The mathematical literature on on phase field systems of Caginalp type is really vast: from the pioneering papers [12,25] and the monography [11] we can count a number of contributions: among them, let us quote $[23,31,32,36,39,41]$. On the other hand, we point out $[6,13,15,20,22,26,27]$ for the analysis of AllenCahn type problems with singular potentials, also including the treatment of 
DISTRIBUTED OPTIMAL CONTROL PROBLEMS

some optimal control problems, and [7, 8, 14, 29, 30, 38] for discussions and results on the Cahn-Hilliard equation with singular potentials. The paper [5] deals with the same Caginalp system (1) in order to investigate sliding mode control problems, whereas the recent contribution [4] is concerned with the conserved phase field model of Caginalp type and focuses on the internal feedback stabilization of the system.

About the optimal control problem, we point out that our concern will be the minimization of the cost functional

$$
\mathcal{\partial}(u):=\frac{1}{2} \int_{Q}(g(\varphi)-\chi)^{2}+\frac{\kappa}{2} \int_{Q}\left(\vartheta-\vartheta_{Q}\right)^{2},
$$

depending on the state variables $\vartheta$ and $\varphi$ subjected to the state system (3)-(6), over all the controls belonging to some control box $\mathcal{U}_{a d}$. The data $\chi$ and $\vartheta_{Q}$ are fixed in $L^{2}(Q), \kappa$ is a nonnegative constant and $g: \mathbb{R} \rightarrow \mathbb{R}$ is a continuous and bounded real function. As for the control box, we simply assume that

$$
\mathcal{U}_{a d}:=\left\{u \in L^{2}(Q): u_{\min } \leq u \leq u_{\max } \text { a.e. in } Q\right\}
$$

where $u_{\min }$ and $u_{\max }$ are given bounded functions. Let us stress here that we can prove the existence of an optimal control for a general class of potentials $\mathcal{W}$, and of course this class includes the potentials $\mathcal{W}_{\text {reg }}, \mathcal{W}_{\text {log }}$ and $\mathcal{W}_{\text {obs }}$ defined in (9)-(11). However, the derivation of the first order necessary optimality conditions can be performed only in case of regular and singular potentials like $\mathcal{W}_{\text {reg }}$ and $\mathcal{W}_{\text {log }}$. Hence, our analyis covers the case of rather general potentials (even singular) in the phase equation and cost functions $\mathcal{J}$ of the form (12).

As far as we know, the contributions on optimal control for Caginalp-type phase field models are quite a few, often restricted to the case of regular potentials or dealing with approximating problems when first order optimality conditions are discussed. In this framework, let us quote the papers $[1,33,34,37]$ and references therein. We also mention the papers [2] for the coupling with Navier-Stokes equations, [3] dealing with a phase relaxation model with double obstacle, [9] for the solification of an alloy, [16] for a boundary control problem with dynamic boundary conditions, $[18,19]$ addressing a nostandard system of phase field equations, [21] for a sharp interface control in a Penrose-Fife system, [24] dealing with an inverse problem for a discontinuous diffusion coefficient, [28] for the study of a damage phase field model in 2D, [40] for a phase field model with total variation functional, [41] for a a class of thermodynamically consistent models: all these contributions are of course involved with distributed or boundary optimal control problems. 
DISTRIBUTED OPTIMAL CONTROL PROBLEMS

\section{The results}

In this section, we state precisely the problem (3)-(6) and introduce our results. Recalling that $\Omega$ is the body where the evolution takes place, we assume $\Omega \subset \mathbb{R}^{3}$ to be a bounded smooth domain with boundary $\Gamma$. Given a final time $T>0$, let

$$
Q:=(0, T) \times \Omega \quad \text { and } \quad \Sigma:=(0, T) \times \Gamma .
$$

Concerning the structure of our system, we assume that

$$
m \in L^{\infty}(\Omega) \text { and } m \geq 0 \text { a.e. in } \Omega \text {, }
$$

$\widehat{\beta}: \mathbb{R} \rightarrow[0,+\infty]$ is convex, proper and l.s.c. with $\widehat{\beta}(0)=0$,

$$
\widehat{\pi}: \mathbb{R} \rightarrow \mathbb{R} \text { is a } C^{1} \text { function and } \pi:=\widehat{\pi}^{\prime} \text { is Lipschitz continuous. }
$$

Setting

$$
\beta:=\partial \widehat{\beta},
$$

we denote by $D(\beta)$ and $D(\widehat{\beta})$ the effective domains of $\beta$ and $\widehat{\beta}$, respectively. In addition, let $\beta^{\circ}(r)$ represent the element of $\beta(r)$ having minimum modulus, for every $r \in D(\beta)$ (see, e.g., [10, p. 28]). In order to simplify notations, we also set

$$
\begin{gathered}
H:=L^{2}(\Omega), \quad V:=H^{1}(\Omega), \\
V_{0}:=H_{0}^{1}(\Omega), \quad W:=\left\{v \in H^{2}(\Omega): \partial_{n} v=0\right\}
\end{gathered}
$$

and endow these spaces with their natural norms. The notation $\|\cdot\|_{X}$ stands for the norm in the generic Banach space $X$, while $\|\cdot\|_{p}$ denotes the usual norm in both $L^{p}(\Omega)$ and $L^{p}(Q)$, for $1 \leq p \leq \infty$. If $v \in L^{2}(0, T ; X)$, we may consider the function $1 * v$ defined by

$$
(1 * v)(t):=\int_{0}^{t} v(s) d s \quad \text { for } t \in[0, T]
$$

(indeed, the symbol $*$ is often employed for convolution products). About the state system, we set the assumptions on the initial data

$$
\begin{gathered}
\vartheta_{0} \in V_{0}, \\
\varphi_{0} \in V \quad \text { and } \widehat{\beta}\left(\varphi_{0}\right) \in L^{1}(\Omega)
\end{gathered}
$$


DISTRIBUTED OPTIMAL CONTROL PROBLEMS

and we look for a triplet $(\vartheta, \varphi, \xi)$ satisfying

$$
\begin{gathered}
\vartheta \in H^{1}(0, T ; H) \cap L^{\infty}\left(0, T ; V_{0}\right) \cap L^{2}\left(0, T ; H^{2}(\Omega)\right), \\
\varphi \in H^{1}(0, T ; H) \cap L^{\infty}(0, T ; V) \cap L^{2}(0, T ; W), \\
\xi \in L^{2}(0, T ; H), \\
\partial_{t} \vartheta-\Delta \vartheta+\partial_{t} \varphi=m u \quad \text { a.e. in } Q, \\
\partial_{t} \varphi-\Delta \varphi+\xi+\pi(\varphi)=\vartheta \quad \text { and } \xi \in \beta(\varphi) \quad \text { a.e. in } Q, \\
\vartheta(0)=\vartheta_{0} \text { and } \varphi(0)=\varphi_{0} \quad \text { a.e. in } \Omega .
\end{gathered}
$$

Note that the boundary conditions explicitly stated in (5) are now hidden in the properties (20) (due to the presence of the space $V_{0}$ ) and (21) (on account of the space $W)$. The above system $(20)-(25)$ is well posed, as stated by the following result.

Theorem 1. Under the assumptions (14)-(16) and (18)-(19), for every $u \in$ $L^{2}(Q)$ the problem $(20)-(25)$ has a unique solution $(\vartheta, \varphi, \xi)$. Moreover, the estimate

$$
\begin{aligned}
& \|\vartheta\|_{H^{1}(0, T ; H) \cap L^{\infty}\left(0, T ; V_{0}\right) \cap L^{2}\left(0, T ; H^{2}(\Omega)\right)} \\
& \quad+\|\varphi\|_{H^{1}(0, T ; H) \cap L^{\infty}(0, T ; V) \cap L^{2}(0, T ; W)}+\|\xi\|_{L^{2}(0, T ; H)} \leq C_{1}
\end{aligned}
$$

holds true for some constant $C_{1}$ that depends only on $\Omega, T$, the structure (14)-(16) of the system, the norms of the initial data in (18)-(19) and $\|u\|_{2}$. Finally, if $u_{i} \in L^{2}(Q), i=1,2$, are given and $\left(\vartheta_{i}, \varphi_{i}, \xi_{i}\right), i=1,2$, denote the respective solutions, then we have

$$
\begin{aligned}
& \left\|\vartheta_{1}-\vartheta_{2}\right\|_{L^{2}(0, T ; H)}+\left\|\left(1 * \vartheta_{1}\right)-\left(1 * \vartheta_{2}\right)\right\|_{L^{\infty}\left(0, T ; V_{0}\right)} \\
& \quad+\left\|\varphi_{1}-\varphi_{2}\right\|_{L^{\infty}(0, T ; H) \cap L^{2}(0, T ; V)} \\
& \leq C^{\prime}\left\|\left(1 * u_{1}\right)-\left(1 * u_{2}\right)\right\|_{L^{2}(0, T ; H)} \leq C^{\prime \prime}\left\|u_{1}-u_{2}\right\|_{L^{2}(0, T ; H)}
\end{aligned}
$$

for some constants $C^{\prime}$ and $C^{\prime \prime}$ depending only on $\Omega, T, \pi$ and $m$.

Some further regularity of the solution follows from the next result.

Theorem 2. Assume (14)-(16) and (18)-(19). Moreover, let

$$
\varphi_{0} \in W \quad \text { and } \quad \beta^{\circ}\left(\varphi_{0}\right) \in H .
$$

Then, the unique solution $(\vartheta, \varphi, \xi)$ provided by Theorem 1 also fulfils

$$
\begin{gathered}
\varphi \in W^{1, \infty}(0, T ; H) \cap H^{1}(0, T ; V) \cap L^{\infty}(0, T ; W), \\
\xi \in L^{\infty}(0, T ; H), \\
\|\varphi\|_{W^{1, \infty}(0, T ; H) \cap H^{1}(0, T ; V) \cap L^{\infty}(0, T ; W)}+\|\xi\|_{L^{\infty}(0, T ; H)} \leq C_{2}, \\
\varphi \in C^{0}(\bar{Q}) \quad \text { and }\|\varphi\|_{\infty} \leq C_{2}
\end{gathered}
$$


DISTRIBUTED OPTIMAL CONTROL PROBLEMS

for some constant $C_{2}$ with the same dependencies as $C_{1}$ plus the norms of the initial data associated to (28). Moreover, If in addition $\vartheta_{0} \in L^{\infty}(\Omega)$ and $u \in L^{\infty}(0, T ; H)$, then there holds

$$
\vartheta \in L^{\infty}(Q) \quad \text { and } \quad\|\vartheta\|_{\infty} \leq C_{3}
$$

for a similar constant $C_{3}$ that depends on $\left\|\vartheta_{0}\right\|_{\infty}$ and $\|u\|_{L^{\infty}(0, T ; H)}$ as well. By further assuming that $\beta^{\circ}\left(\varphi_{0}\right) \in L^{\infty}(\Omega)$, it turns out that $\xi \in L^{\infty}(Q)$ and

$$
\|\xi\|_{L^{\infty}(Q)} \leq C_{4}
$$

where the constant $C_{4}$ also depends on $C_{3}$ and $\left\|\beta^{\circ}\left(\varphi_{0}\right)\right\|_{\infty}$.

In view of the above results, we can now specify the control-to-state mapping $\mathcal{S}$ and introduce the corresponding control problem. Let

$$
\begin{aligned}
& x:=L^{\infty}(Q), \\
& y:=y_{1} \times y_{2} \quad \text { where } \quad y_{1}:=\left\{v \in L^{2}(Q): 1 * v \in L^{2}\left(0, T ; V_{0}\right)\right\} \\
& \quad \text { and } y_{2}:=L^{\infty}(0, T ; H) \cap L^{2}(0, T ; V), \\
& \mathcal{S}: X \rightarrow y, \quad u \mapsto \mathcal{S}(u)=:(\vartheta, \varphi) \text { where } \\
& \quad(\vartheta, \varphi, \xi) \text { is the unique solution to }(20)-(25) \text { corresponding to } u .
\end{aligned}
$$

We also want to give a precise definition of the control box and of the cost functional. To this aim, we suppose that

$$
\begin{gathered}
u_{\min }, u_{\max } \in L^{\infty}(Q) \quad \text { satisfy } \quad u_{\min } \leq u_{\max } \text { a.e. in } Q \\
g: \mathbb{R} \rightarrow \mathbb{R} \text { is continuous and bounded } \\
\kappa \in[0,+\infty) \text { and } \chi, \vartheta_{Q} \in L^{2}(Q)
\end{gathered}
$$

and, in view of (13) and (12), we recall that

$$
\begin{aligned}
& \mathcal{U}_{a d}:=\{u \in \mathcal{X}:\left.u_{\min } \leq u \leq u_{\max } \text { a.e. in } Q\right\} \\
& \mathcal{J}:=\mathcal{F} \circ \mathcal{S}: \mathcal{X} \rightarrow \mathbb{R} \text { where } \mathcal{F}: \mathcal{Y} \rightarrow \mathbb{R} \text { is defined by } \\
& \mathcal{F}(\vartheta, \varphi):=\frac{1}{2} \int_{Q}(g(\varphi)-\chi)^{2}+\frac{\kappa}{2} \int_{Q}\left(\vartheta-\vartheta_{Q}\right)^{2} .
\end{aligned}
$$

The first result on the control problem is stated below.

Theorem 3. Under the assumptions (14)-(16) and (18)-(19), let $\mathcal{U}_{\text {ad }}$ and $\mathrm{J}$ be defined by (41)-(42). Then, there exists $u^{*} \in \mathcal{U}_{\text {ad }}$ such that

$$
\mathcal{J}\left(u^{*}\right) \leq \mathcal{J}(u) \quad \text { for every } u \in \mathcal{U}_{\text {ad }} .
$$


DISTRIBUTED OPTIMAL CONTROL PROBLEMS

From now on, the assumptions (14)-(16) as well as those on the structure and on the initial data are in force. Our aim is formulating the first order necessary optimality conditions: as $\mathcal{U}_{a d}$ is convex, the desired necessary condition for optimality is

$$
\left\langle D \mathcal{J}\left(u^{*}\right), u-u^{*}\right\rangle \geq 0 \quad \text { for every } u \in \mathcal{U}_{a d}
$$

provided that the derivative $D \mathcal{J}\left(u^{*}\right)$ exists in the dual space $X^{*}$ at least in the Gâteaux sense. Thus, the natural approach leads us to check whether $\mathcal{S}$ is Fréchet differentiable at $u^{*}$ and apply the chain rule to $\mathcal{J}=\mathcal{F} \circ \mathcal{S}$. In order to carry out this program, we need further assumptions on the nonlinearities $\beta$, $\pi$ and $g$. Namely, we also assume

$$
\begin{gathered}
D(\beta) \text { is an open interval and } \beta \text { is single-valued on } D(\beta), \\
\beta \text { and } \pi \text { are } C^{2} \text { functions and } g \text { is a } C^{1} \text { function. }
\end{gathered}
$$

We remark that (45) implies $\beta^{\circ}=\beta$. Moreover, the inclusion in (24) becomes $\xi=\beta(\varphi)$ and now $\beta$ and $\pi$ enter the problem through their sum, mainly. Hence, for brevity we can set

$$
\gamma:=\beta+\pi
$$

and $\gamma$ turns out to be a $C^{2}$ function on $D(\beta)$. We also observe that the functions $\beta$ and $\pi$ resulting from the derivatives of the potentials $\mathcal{W}_{\text {reg }}$ in (9) and $\mathcal{W}_{l o g}$ in (10), both comply with (45)-(46). Another choice of an admissible nonlinearity $\beta$ is given by

$$
\beta(r):=1-\frac{1}{r+1} \quad \text { for } r>-1
$$

and it corresponds to the convex function

$$
\widehat{\beta}(r):= \begin{cases}r-\ln (r+1) & \text { if } r>-1 \\ +\infty & \text { otherwise }\end{cases}
$$

taking the minimum value 0 at 0 , as required in the assumption (15). This choice of $\beta$ yields an example of a different behavior for negative and positive values, singular near -1 and with a bounded growth at $+\infty$.

Next, note that assumptions (45)-(46) and definition (47) force $\beta(r)$ and consequently $\gamma(r)$ to tend to $\pm \infty$ as $r$ tends to a finite end-point of $D(\beta)$, if any. Hence, combining (45)-(46) with the boundedness of $\varphi$ and $\xi$ given by Theorem 2, it is straightforward to infer the following result. 
DISTRIBUTED OPTIMAL CONTROL PROBLEMS

Corollary 4. Under all the assumptions used in Theorem 2, let (45)-(46) hold true, in addition. Then, the component $\varphi$ of the solution $(\vartheta, \varphi, \xi)$ also satisfies

$$
\varphi_{\mathrm{inf}} \leq \varphi \leq \varphi_{\mathrm{sup}} \quad \text { in } \bar{Q}
$$

where $\varphi_{\mathrm{inf}}, \varphi_{\mathrm{sup}}$ are constants lying in $D(\beta)$ and depending only on $\Omega, T$, the structure (14)-(16) and (45)-(46) of the system, the norms of the initial data associated to (18)-(19), and the norms $\|u\|_{\infty},\left\|\vartheta_{0}\right\|_{\infty}$ and $\left\|\beta\left(\varphi_{0}\right)\right\|_{\infty}$.

As already announced, we aim to compute the Fréchet derivative of $\mathcal{S}$. Then, we have to consider the linearized problem described below, which can be stated starting from a generic element $\bar{u} \in X$.

Let $\bar{u} \in \mathcal{X}, h \in \mathcal{X}$ be given and set $(\bar{\vartheta}, \bar{\varphi}):=\mathcal{S}(\bar{u})$. We are interested to find a pair $(\Theta, \Phi)$ satisfying

$$
\begin{gathered}
\Theta \in H^{1}(0, T ; H) \cap L^{\infty}\left(0, T ; V_{0}\right) \cap L^{2}\left(0, T ; H^{2}(\Omega)\right), \\
\Phi \in H^{1}(0, T ; H) \cap L^{\infty}(0, T ; V) \cap L^{2}(0, T ; W)
\end{gathered}
$$

and solving the following problem

$$
\begin{array}{cl}
\partial_{t} \Theta-\Delta \Theta+\partial_{t} \Phi=m h & \text { a.e. in } Q, \\
\partial_{t} \Phi-\Delta \Phi+\gamma^{\prime}(\bar{\varphi}) \Phi=\Theta & \text { a.e. in } Q, \\
\Theta(0)=0 \text { and } \quad \Phi(0)=0 & \text { a.e. in } \Omega .
\end{array}
$$

We can repeat here the remark concerning the boundary conditions for $\Theta$ and $\Phi$ : these boundary conditions are contained in (49) and (50).

Proposition 5. Let $\bar{u} \in \mathcal{X}$ and $(\bar{\vartheta}, \bar{\varphi})=\mathcal{S}(\bar{u})$. Then, for every $h \in X$, there is a unique pair $(\Theta, \Phi)$ solving the linearized problem (49)-(53). Moreover, the inequality

$$
\|(\Theta, \Phi)\|_{y} \leq C_{5}\|h\|_{x}
$$

holds true for some constant $C_{5}$ depending only on $\Omega, T$, the structure (14)(16) and (45)-(46) of the system, the norms of the initial data associated to (18)-(19), and the norms $\|\bar{u}\|_{\infty},\left\|\vartheta_{0}\right\|_{\infty}$ and $\left\|\beta\left(\varphi_{0}\right)\right\|_{\infty}$. In particular, the linear map $\mathcal{D}: h \mapsto(\Theta, \Phi)$ is continuous from $\mathcal{X}$ to $\mathcal{y}$.

Therefore, the Fréchet derivative $D \mathcal{S}(\bar{u}) \in \mathcal{L}(X, y)$ actually exists and coincides with the map $\mathcal{D}$ introduced in Proposition 5. This property being established, it is possible to exploit the chain rule with $\bar{u}:=u^{*}$ to show that the necessary condition (44) for optimality takes the form

$$
\int_{Q}\left(g\left(\varphi^{*}\right)-\chi\right) g^{\prime}\left(\varphi^{*}\right) \Phi+\kappa \int_{Q}\left(\vartheta^{*}-\vartheta_{Q}\right) \Theta \geq 0 \quad \text { for all } u \in \mathcal{U}_{a d}
$$


DISTRIBUTED OPTIMAL CONTROL PROBLEMS

where $\left(\vartheta^{*}, \varphi^{*}\right)=\mathcal{S}\left(u^{*}\right)$ and, for a given $u \in \mathcal{U}_{a d}$, the pair $(\Theta, \Phi)$ is exacly the solution to the linearized problem (49)-(53) corresponding to $h=u-u^{*}$.

The final step then consists in eliminating the pair $(\Theta, \Phi)$ from (55). This will be done by introducing a pair $(p, q)$ solving the adjoint problem, that is, fulfilling the regularity requirements

$$
\begin{gathered}
p \in H^{1}(0, T ; H) \cap L^{\infty}\left(0, T ; V_{0}\right) \cap L^{2}\left(0, T ; H^{2}(\Omega)\right), \\
q \in H^{1}(0, T ; H) \cap L^{\infty}(0, T ; V) \cap L^{2}(0, T ; W)
\end{gathered}
$$

and satisfying

$$
\begin{array}{cc}
-\partial_{t} p-\Delta p-q=\kappa\left(\vartheta^{*}-\vartheta_{Q}\right) & \text { a.e. in } Q, \\
-\partial_{t} q-\Delta q+\gamma^{\prime}\left(\varphi^{*}\right) q-\partial_{t} p=\left(g\left(\varphi^{*}\right)-\chi\right) g^{\prime}\left(\varphi^{*}\right) & \text { a.e. in } Q, \\
p(T)=q(T)=0 & \text { a.e. in } \Omega
\end{array}
$$

Recalling the definition of the spaces $V_{0}$ and $W$, once more we point out that, as in previous cases (compare with (20)-(25) and (49)-(53)), the Dirichlet boundary condition for $p$ is included in (56) whereas the Neumann boundary condition for $q$ is in (57).

Theorem 6. Let $u^{*}$ be an optimal control and let $\left(\vartheta^{*}, \varphi^{*}\right)=\mathcal{S}\left(u^{*}\right)$ denote the corresponding state. Then there existes a unique solution $(p, q)$ of the adjoint problem (56)-(60).

The last statement regards the optimality conditions.

Theorem 7. Let $u^{*}$ be an optimal control. Moreover, let $\left(\vartheta^{*}, \varphi^{*}\right)=\mathcal{S}\left(u^{*}\right)$ and $(p, q)$ be the associate state and the unique solution to the adjoint problem (58)(60) given by Theorem 6. Then there holds

$$
\begin{array}{r}
m(x) p(t, x)\left(u-u^{*}(t, x)\right) \geq 0 \quad \text { for every } u \in\left[u_{\min }(t, x), u_{\max }(t, x)\right], \\
\text { for a.a. }(t, x) \in Q .
\end{array}
$$

In particular, we have that $m p=0$ in the subset of $Q$ where $u_{\min }<u^{*}<u_{\max }$.

An easy consequence of Theorem 7 is the following.

Corollary 8. Under the conditions of Theorem 7, the optimal control $u^{*}$ fulfills

$$
u^{*} \begin{cases}=u_{\min } & \text { a.e. } \text { in the subset of } Q \text { where } p>0 \text { and } m>0 \\ =u_{\max } & \text { a.e. in the subset of } Q \text { where } p<0 \text { and } m>0 \\ \text { is undetermined } & \text { elsewhere. }\end{cases}
$$


DISTRIBUTED OPTIMAL CONTROL PROBLEMS

All the results stated in this section are rigorously proved in the paper [17], to which we refer for details and proofs. As a final remark, let us point out that it would be interesting to extend the above results to the conserved phase field model of Caginalp type, in which the equation for the phase variable $\varphi$ is replaced by the system

$$
\partial_{t} \varphi-\Delta \mu=0, \quad \mu=-\Delta \varphi+\beta(\varphi)+\pi(\varphi)-\vartheta \quad \text { in } Q,
$$

and the additional variable $\mu$, termed chemical potential, should also satisfy a no-flux boundary condition in order that the mean value of $\varphi$ be conserved. The resulting system turns out to be a model for phase separaration in binary mixtures, in particular, and it is related to Cahn-Hilliard equations and systems (see, e.g., [4] and reference therein).

\section{Acknowledgements}

This research activity has been performed in the framework of an ItalianRomanian three-year project on "Control and stabilization problems for phase field and biological systems" financed by the Italian CNR and the Romanian Academy. Moreover, some financial support from the FP7-IDEAS-ERC-StG \#256872 (EntroPhase), the MIUR-PRIN Grant 2015PA5MP7 "Calculus of Variations" and the project Fondazione Cariplo-Regione Lombardia MEGAsTAR "Matematica d'Eccellenza in biologia ed ingegneria come accelleratore di una nuona strateGia per l'ATtRattività dell'ateneo pavese" is gratefully acknowledged by the authors. Finally, the present paper also benefits from the support of the GNAMPA (Gruppo Nazionale per l'Analisi Matematica, la Probabilità e le loro Applicazioni) of INdAM (Istituto Nazionale di Alta Matematica) for PC, GG and ER.

\section{References}

[1] F.D. Araruna, J.L. Boldrini, B.M.R. Calsavara, Optimal control and controllability of a phase field system with one control force. Appl. Math. Optim. 70 (2014), 539-563.

[2] L'. Baňas, M. Klein, A. Prohl, Control of interface evolution in multiphase fluid flows. SIAM J. Control Optim. 52 (2014), 2284-2318.

[3] V. Barbu, M.L. Bernardi, P. Colli, G. Gilardi, Optimal control problems of phase relaxation models, J. Optim. Theory Appl. 109 (2001), 557-585. 
DISTRIBUTED OPTIMAL CONTROL PROBLEMS

[4] V. Barbu, P. Colli, G. Gilardi, G. Marinoschi, Feedback stabilization of the Cahn-Hilliard type system for phase separation, J. Differential Equations 262 (2017), 2286-2334

[5] V. Barbu, P. Colli, G. Gilardi, G. Marinoschi, E. Rocca, Sliding mode control for a nonlinear phase-field system, SIAM J. Control Optim, to appear in 2017 (see also preprint arXiv:1506.01665 [math.AP] (2015), pp. 1-28).

[6] T. Benincasa, L.D. Donado Escobar, C. Moroşanu, Distributed and boundary optimal control of the Allen-Cahn equation with regular potential and dynamic boundary conditions. Internat. J. Control 89 (2016), 1523-1532.

[7] J.F. Blowey, C.M. Elliott, The Cahn-Hilliard gradient theory for phase separation with non-smooth free energy. I. Mathematical Analysis, European J. Appl. Math. 2 (1991), 233-280.

[8] J.F. Blowey, C.M. Elliott, The Cahn-Hilliard gradient theory for phase separation with nonsmooth free energy. II. Numerical Analysis, European J. Appl. Math. 3 (1992), 147-179.

[9] J.L. Boldrini, B.M.C. Caretta, E. Fernández-Cara, Some optimal control problems for a two-phase field model of solidification, Rev. Mat. Complut. 23 (2010), 49-75.

[10] H. Brezis, "Opérateurs maximaux monotones et semi-groupes de contractions dans les espaces de Hilbert", North-Holland Math. Stud. 5, North-Holland, Amsterdam, 1973.

[11] M. Brokate, J. Sprekels, "Hysteresis and Phase Transitions", Springer, New York, 1996.

[12] G. Caginalp, An analysis of a phase field model of a free boundary, Arch. Rational Mech. Anal 92 (1986), 205-245.

[13] L. Calatroni, P. Colli, Global solution to the Allen-Cahn equation with singular potentials and dynamic boundary conditions, Nonlinear Anal. 79 (2013), 12-27.

[14] L. Cherfils, A. Miranville, S. Zelik, The Cahn-Hilliard equation with logarithmic potentials, Milan J. Math. 79 (2011), 561-596.

[15] P. Colli, M.H. Farshbaf-Shaker, J. Sprekels, A deep quench approach to the optimal control of an Allen-Cahn equation with dynamic boundary conditions and double obstacles, Appl. Math. Optim. 71 (2015), 1-24. 
DISTRIBUTED OPTIMAL CONTROL PROBLEMS

[16] P. Colli, G. Gilardi, G. Marinoschi, A boundary control problem for a possibly singular phase field system with dynamic boundary conditions, J. Math. Anal. Appl. 434 (2016), 432-463.

[17] P. Colli, G. Gilardi, G. Marinoschi, E. Rocca, Optimal control for a phase field system with a possibly singular potential, Math. Control Relat. Fields 6 (2016), 95-112.

[18] P. Colli, , G. Gilardi, P. Podio-Guidugli, J. Sprekels, Distributed optimal control of a nonstandard system of phase field equations, Contin. Mech. Thermodyn. 24 (2012), 437-459.

[19] P. Colli, G. Gilardi, J. Sprekels, Analysis and optimal boundary control of a nonstandard system of phase field equations, Milan J. Math. 80 (2012), 119-149.

[20] P. Colli, G. Gilardi, J. Sprekels, A boundary control problem for the pure Cahn-Hilliard equation with dynamic boundary conditions, Adv. Nonlinear Anal. 4 (2015), 311-325.

[21] P. Colli, G. Marinoschi, E. Rocca, Sharp interface control in a PenroseFife model, ESAIM Control Optim. Calc. Var. 22 (2016), 473-499.

[22] P. Colli, J. Sprekels, Optimal control of an Allen-Cahn equation with singular potentials and dynamic boundary condition, SIAM J. Control Optim. 53 (2015), 213-234.

[23] A. Damlamian, N. Kenmochi, N. Sato, Subdifferential operator approach to a class of nonlinear systems for Stefan problems with phase relaxation, Nonlinear Anal. 23 (1994), 115-142.

[24] K. Deckelnick, C.M. Elliott, V. Styles, Double obstacle phase field approach to an inverse problem for a discontinuous diffusion coefficient. Inverse Problems 32 (2016), 045008, 26 pp.

[25] C.M. Elliott, S. Zheng, Global existence and stability of solutions to the phase-field equations, in "Free boundary problems", Internat. Ser. Numer. Math., 95, 46-58, Birkhäuser Verlag, Basel, (1990).

[26] M.H. Farshbaf-Shaker, A penalty approach to optimal control of AllenCahn variational inequalities: MPEC-view, Numer. Funct. Anal. Optim. 33 (2012), 1321-1349.

[27] M.H. Farshbaf-Shaker, C. Hecht, Optimal control of elastic vector-valued Allen-Cahn variational inequalities, SIAM J. Control Optim. 54 (2016), 129-152. 
DISTRIBUTED OPTIMAL CONTROL PROBLEMS

FOR PHASE FIELD SYSTEMS WITH SINGULAR POTENTIAL

[28] M.H. Farshbaf-Shaker, C. Heinemann, A phase field approach for optimal boundary control of damage processes in two-dimensional viscoelastic media. Math. Models Methods Appl. Sci. 25 (2015), 2749-2793.

[29] G. Gilardi, A. Miranville, G. Schimperna, On the Cahn-Hilliard equation with irregular potentials and dynamic boundary conditions, Commun. Pure Appl. Anal. 8 (2009), 881-912.

[30] G. Gilardi, A. Miranville, G. Schimperna, Long-time behavior of the Cahn-Hilliard equation with irregular potentials and dynamic boundary conditions, Chin. Ann. Math. Ser. B 31 (2010), 679-712.

[31] M. Grasselli, A. Miranville, G. Schimperna, The Caginalp phase-field system with coupled dynamic boundary conditions and singular potentials, Discrete Contin. Dyn. Syst. 28 (2010), 67-98.

[32] M. Grasselli, H. Petzeltová, G. Schimperna, Long time behavior of solutions to the Caginalp system with singular potential, Z. Anal. Anwend. 25 (2006), 51-72.

[33] K.-H. Hoffmann, L.S. Jiang, Optimal control of a phase field model for solidification, Numer. Funct. Anal. Optim. 13 (1992), 11-27.

[34] K.-H. Hoffmann, N. Kenmochi, M. Kubo, N. Yamazaki, Optimal control problems for models of phase-field type with hysteresis of play operator, Adv. Math. Sci. Appl. 17 (2007), 305-336.

[35] N. Kenmochi, M. Niezgódka, Evolution systems of nonlinear variational inequalities arising phase change problems, Nonlinear Anal. 22 (1994), 1163-1180.

[36] Ph. Laurençot, Long-time behaviour for a model of phase-field type, Proc. Roy. Soc. Edinburgh Sect. A 126 (1996), 167-185.

[37] C. Lefter, J. Sprekels, Optimal boundary control of a phase field system modeling nonisothermal phase transitions, Adv. Math. Sci. Appl. 17 (2007), 181-194.

[38] A. Miranville, S. Zelik, Robust exponential attractors for Cahn-Hilliard type equations with singular potentials, Math. Methods Appl. Sci. 27 (2004), 545-582.

[39] G. Schimperna, Abstract approach to evolution equations of phase field type and applications, J. Differential Equations 164 (2000), 395-430. 
DISTRIBUTED OPTIMAL CONTROL PROBLEMS

[40] K. Shirakawa, N. Yamazaki, Optimal control problems of phase field system with total variation functional as the interfacial energy, Adv. Differential Equations 18 (2013), 309-350.

[41] J. Sprekels, S. Zheng, Optimal control problems for a thermodynamically consistent model of phase-field type for phase transitions, Adv. Math. Sci. Appl. 1 (1992), 113-125.

Pierluigi Colli

Dipartimento di Matematica "F. Casorati"

Università di Pavia

and IMATI-CNR, Pavia

via Ferrata 1, 27100 Pavia, Italy

e-mail: gianni.gilardi@unipv.it

Gianni Gilardi

Dipartimento di Matematica "F. Casorati"

Università di Pavia

and IMATI-CNR, Pavia

via Ferrata 1, 27100 Pavia, Italy

e-mail: gianni.gilardi@unipv.it

Gabriela Marinoschi

"Gheorghe Mihoc-Caius Iacob" Institute

of Mathematical Statistics and Applied

Mathematics

of the Romanian Academy (ISMMA)

Calea 13 Septembrie 13, 050711 Bucharest,

Romania

e-mail: gabriela.marinoschi@acad.ro

Elisabetta Rocca

Dipartimento di Matematica "F. Casorati"

Università di Pavia

and IMATI-CNR, Pavia

via Ferrata 1, 27100 Pavia, Italy

e-mail: elisabetta.rocca@unipv.it 